\title{
Es war 4 mal - Érase 4 veces: Ein Theaterprojekt im DaF-Unterricht der Deutschen Schule Valdivia
}

\author{
Daniel Berghoff
}

\begin{abstract}
Zusammenfassung
Um die Siebtklässler der Deutschen Schule Valdivia in Chile zum Deutschlernen zu motivieren, wurden im Schuljahr 2013 zwei der insgesamt sechs wöchentlichen Deutsch als Fremdsprache (DaF)-Stunden erstmalig als Theaterkurs angeboten. Ziel des Pilotprojektes war es, die mündliche Kommunikation der Schülerinnen und Schüler ${ }^{1}$ zu verbessern und gleichzeitig ihre kreativen Ausdruckfähigkeiten zu fördern. Alle Schüler der Jahrgangsstufe erhielten so die Möglichkeit, Deutsch auf eine kreative Art zu lernen und vor allem in der Praxis anzuwenden. Im ersten Schulhalbjahr wurden zunächst viele verschiedene Bereiche des Theaters spielerisch thematisiert. Der Theaterunterricht aller drei Lerngruppen fand natürlich auf Deutsch statt, so dass einerseits das Hörverständnis der Schüler gefördert wurde, andererseits durch die eigenständige Entwicklung der späteren Dialoge für eine Aufführung die Sprachpraxis geübt wurde. Als Thema wurde das Märchen von Schneewittchen gewählt, welches von den drei Gruppen verfremdet werden sollte. So entstanden drei kurze, moderne Versionen auf Deutsch. Eine vierte Version wurde von der spanischsprachigen Theater-AG entwickelt. Die beiden Theaterlehrer führten als Jakob und Wilhelm Grimm durch den Abend und bereiteten das nicht-deutschsprachige Publikum bilingual auf Spanisch und Deutsch auf die Handlung vor. Der Erfolg des Pilotprojekts führte dazu, dass Theater im DaF-Unterricht an der Schule fest im Curriculum verankert wurde. Im Artikel wird erläutert, wie auch an anderen Schulen nach ähnlichem Schema, Theater im DaF-Unterricht die mündliche Kommunikation fördern und stärken kann.
\end{abstract}

\section{Gedanken zum Schultheater}

Viele der Theaterstücke, die in der Schule aufgeführt werden, sind der Mehrheit des Publikums bekannt. Sei es, weil es die typischen Klassiker sind oder weil sich Literaturkurse mit einem Drama beschäftigen (müssen), da es zum Literatur-Kanon innerhalb der Abiturvorgaben gehört oder weil es

\footnotetext{
${ }^{1}$ Im Artikel wird im Weiteren eine einheitliche Form verwendet, um den Lesefluss nicht zu sehr zu beeinträchtigen. Natürlich schließt diese immer die männliche und weibliche Form ein.
} 
gar der Unkreativität bzw. der Einfallslosigkeit der Theaterlehrer geschuldet ist. Doch will man eine gute Inszenierung eines bekannten Dramas sehen, geht man doch eigentlich in die Aufführung eines professionellen Theaters, da sich hier Personen mit der Umsetzung eines Theaterstückes beschäftigen, die dies gelernt haben und man eine vernünftige Interpretation des Stoffes erwarten kann. Im Bereich Schule und Theater oder gar Theater im Unterricht haben wir es bei den Akteuren, also den Lernenden, selten mit Personen zu tun, die sich beruflich mit dem Bereich Theater auseinandersetzen bzw. dies später einmal vorhaben. Somit kommt Theater in der Schule ein Sonderstatus zu, der gewisse Anforderungen an die Theaterschaffenden (in erster Linie an die Pädagogen) stellt, besonders wenn auch diese sich noch nie professionell mit Theater(pädagogik) auseinandergesetzt haben. Wie also soll Schultheater die Erwartungen des Publikums an eine innovative Umsetzung einer bekannten Dramenvorlage erfüllen. Dies gelingt meist nur, wenn man den Bewertungsmaßstab senkt, was sich dann oft im Anschluss an eine Schultheateraufführung in Äußerungen wie Für Schultheater war es gut! zeigt. Doch dieser Satz ist eigentlich nicht notwendig, wenn sich Theaterlehrer mehr als Anleiter oder Spielleiter verstehen, die den Schülern Möglichkeiten anbieten, selbst kreativ zu werden. Das bekannte Theaterstück kann dabei ja trotzdem noch Pate stehen, aber es sollte nicht für einen Versuch der Nachahmung von Profiensembles verkommen. Dabei werden wichtige Möglichkeiten verschenkt und der eigentliche Auftrag von Schultheater nicht erfüllt, nämlich der Bildungsauftrag, den Schule und somit jedes Schulfach (also auch Theater) haben. Theaterlehrer, besonders Anfänger, müssen ermutigt werden, Theater in der Schule in Form einer Theaterpädagogik zu begreifen und zwar mit dem Fokus auf dem zweiten Teil des Kompositums. Möglichkeiten dazu gibt es genug und die Erwartungen der Kollegen und Eltern können sicher auch damit erfüllt werden, besonders wenn man mit ihnen über den Sinn von Theaterarbeit in der Schule redet.

Ziel des Schultheaters ist nämlich, wie oben erwähnt, die Bildung der Schüler und nicht die Kunst (vgl. Liebau 2013: 38). Jedoch geht es um eine künstlerische Bildung, demnach müssen die Kreativität und die Kunstfrömmigkeit zentrale Aspekte des Schultheaters sein. "Wichtig dabei ist, dass es sich hier nicht vorrangig um die Beschäftigung mit dem künstlerisch Theatralen handelt, sondern dass das Spiel als Mittel zur Eigenerfahrung begriffen wird. Der Prozess steht im Mittelpunkt, nicht das Produkt; das Ausprobieren, nicht das Gelingen."(Hoffmann 2008: 206)

Die Schüler sollen also angeleitet werden, Kompetenzen (z. B. im ästhetischen Ausdruck) zu entwickeln, sich selbst zu erfahren und Möglichkeiten in der Darstellung (vor dem Publikum) zu erproben und zu erfahren. Dazu müssen sowohl positive als auch negative Erfahrungen gehören, um eine Entwicklung im Sinne einer Verbesserung zu durchlaufen. Nicht das Stück (das Produkt oder die Produktion) steht im Vordergrund, sondern die Kreativität - also der (Lern)prozess auf dem Weg zur Produktion, denn dabei lernen und erfahren die Schüler viel über sich selbst und werden gebildet. Es kann also nicht darum 
gehen, ein vorhandenes Drama (oder eine andere Textvorlage) zu reproduzieren im Sinne einer Verteilung von Rollen, Text lernen und Wiedergabe des Textes auf der Bühne in bestimmter Rollen- und Bühnenanordnung - wie oftmals besonders von Deutschlehrern ohne Erfahrung im Bereich des Darstellenden Spiels gedacht und von den Eltern erwartet. Falls es jedoch genau zu dieser Reproduktion kommt, kommt es leider auch meist nur zum Äufsagtheater"(vgl. Meisenberg 2013: 14). Ich stimme sowohl Meisenberg als auch Tina Hoffmann zu, die zum Aufsagtheater in Bezug auf den Fremdsprachenunterricht folgender Meinung ist:

Es ist ein verbreiteter Irrtum, dass die Produktion eines Theaterstücks mit Schülern oder Studierenden mit einer gründlichen Textanalyse und dem anschließenden Lernen des Textes beginnen müsse. Bei der Theaterarbeit mit Studierenden geschieht nicht selten Folgendes: Einer der Mitwirkenden an der Inszenierung hat nach den Leseproben seine ersten Textpassagen fleißig auswendig gelernt. Bei den ersten Proben auf der Bühne möchte er nun wissen, wo er wann seinen Text aufsagen soll. Es entsteht ein statisches Rezitieren des gut gelernten Textes, der mit dem Körper nicht nachempfunden wird und so weder für den Zuschauer noch für den Darsteller selbst emotional nachvollziehbar ist. Damit sich der Darsteller den Text zu eigen machen kann, damit er ihn leben kann, auch in der Fremdsprache, bedarf es m. E. immer einer Textproduktion im Kontext einer Handlung. Nur so kann der Darsteller den fremdsprachigen Text als Ausdruck seiner „eigenen“ Gedanken und Emotionen begreifen. (Hoffmann 2008: 206)

In Bezug auf Theater im Unterricht des Faches Deutsch als Fremdsprache (DaF), hat Birgit Oelschläger einen interessanten Aufsatz verfasst, aus dem im Folgenden zitiert werden soll:

Eines der Hauptargumente, die oft gegen Theater-Spielen im DaF-Unterricht genannt werden, ist das mangelnde Sprachniveau der Schüler. Dabei wird davon ausgegangen, dass im Unterricht ein komplexes Theaterstück inszeniert wird, oftmals ein bekanntes Drama der deutschen Literatur, das dann wortgetreu in Szene gesetzt wird. Die szenische Improvisation, bei der Texte nur als Ausgangsbasis für persönliche und spontane Darbietungen dienen, erscheint den meisten Lehrern als eine Utopie, denn „meine Schüler bekommen ja sowieso kaum den Mund auf“. Und dennoch ist es selbst auf Anfängerniveau sehr wohl möglich, Elemente des Theaters einzusetzen. (Oelschläger 2004: 25)

Weiter heißt es bei Oelschläger:

Szenisches Spiel im DaF-Unterricht heißt nicht „wir spielen 'Faust' und haben viel Spaß", sondern wie stets im Unterricht steht die Frage nach dem Ziel im Vordergrund. Was kann durch diese oder jene Übung oder Improvisation erreicht werden? Des Weiteren: Wie gehen wir mit dem Wortschatz um, wie kann in das Thema eingestiegen werden, wie bauen die Unterrichtsschritte aufeinander auf? Undsoweiter. (Oelschläger 2004: 33) 
Natürlich sollte es generell immer eine kreative Auseinandersetzung mit einem Thema geben. Dieses Thema kann freilich einem Drama (oder einem sonstigen Text) entstammen, wie es im Schultheater auch meist der Alltag ist (vgl. Studt 2013: 6). Die wichtige, kreative Arbeit beginnt jedoch erst bei und mit der Adaptation und setzt sich dann in der Ausarbeitung einer eigenen Version (des Stückes) fort. Hier muss der Theaterlehrer als Coach zur Seite stehen, der teilweise die Schüler berät, Möglichkeiten anbietet, aber - wenn es sein muss den Arbeitsprozess auch in die richtige Richtung (damit ist eine gemeinsame, für die Gruppe umsetzbare Lösung gemeint) lenkt. Für die Arbeit ist es zunächst wichtig, dass die Gruppe zum Einen zur Gruppe wird und sich als Team begreift, das nur gemeinsam eine gute Arbeit leisten kann. (Starallüren sind hier genauso wenig gefragt wie ein völliges Zurücknehmen und die Anderen machen lassen bzw. ein Konsumieren.) Das heißt für die praktische Arbeit, dass am Anfang einer jeden schulischen Theaterarbeit der Fokus auf der Gruppenbildung liegen sollte, die z. B. durch Sensibilisierungs- und/oder Vertrauensübungen erreicht werden kann. Zum Anderen müssen die Schüler mit Theater (also mit Darstellung, Kreativität, Ausdruck usw.) vertraut werden, besonders wenn es sich in erster Linie um Anfänger handelt. Die Schüler benötigen einen Erfahrungsschatz hinsichtlich verschiedener Ausdrucksmöglichkeiten für eigene Produktionen bzw. Präsentationen (z. B. Tanztheater, Pantomime, Choreografie, Musiktheater, Standbilder, Rhythmus, Atem und Stimme, Improvisation usw.).

Das Einfangen des Publikums und damit ein Erfolg auf der Bühne sind nur möglich, wenn der Ausdruck in Bewegung und Stimme ganzheitlich ist, d.h. wenn die Spielhaltung einer Figur gefunden wurde. Dazu bedarf es sehr viel Grundlagenarbeit. Das Grundspektrum erlebbarer Gefühle, das jeder Mensch in sich trägt, muss für die Bühne verfügbar gemacht werden. Bewegungs-, Atem- und Stimmübungen helfen dabei, sich dieses Spektrums bewusst zu werden bzw. dieses auf der Bühne abrufbar zu machen. (Hoffmann 2008: 209)

Erst nachdem diese beiden grundlegenden Prozesse abgeschlossen oder zumindest fortgeschritten sind, können Ideen für eine mögliche (jedoch nicht zwingende) Aufführung gesammelt und entwickelt werden. Hierbei ist es natürlich am besten und schönsten, wenn das Thema, mit dem sich die Gruppe (szenisch) auseinandersetzt aus der Gruppe selbst entsteht, z. B. aus aktuellen Anlässen, aus gemeinsamen Interessen aber auch aus entstandenen Konflikten oder Problemen (in der Gruppe oder mit dem Lehrer bzw. im schulischen Kontext). Somit kann die Theaterarbeit - besonders bei Schülern in der Pubertät - ein wichtiges Instrument beim Erwachsenwerden oder im Selbstfindungsprozess und auf dem Weg zur Herausbildung einer eigenen Rolle innerhalb sozialer Bezüge werden. Natürlich können bei der Arbeit mit einem Thema wie bereits erwähnt literarische Vorlagen einfließen. Das Anbieten von (weiteren) Möglichkeiten und das Angebot zur Auseinandersetzung mit den Gedanken anderer Menschen (also Autoren) obliegt dem Lehrer, der damit auch einen wichtigen Auftrag erfüllt und Schülern Literatur auf eine andere Art und Weise (im Sinne der literacy-Erziehung) nahe bringen kann. 
Ein wichtiger Hinweis für den Arbeitsprozess ist die Offenhaltung der Rollenverteilung, die erst zu einem späten Zeitpunkt erfolgen sollte, damit die Gesamtgruppe so lange wie möglich ihre gesamte Kreativität in die Dramaturgie und die Herausarbeitung der Figuren(konstellation) stecken kann. Wird zu früh mit der Vergabe der Rollen begonnen, denken viele der Schüler bei der weiteren kreativen Arbeit eher an die einzelnen Darsteller und nicht an die zu verkörpernden Figuren oder beschäftigen sich zu sehr mit der eigenen Rolle, so dass u. U. wichtiges kreatives Potential nicht genutzt werden kann. Es besteht also die Gefahr, dass sie bei der Ausarbeitung der Charaktere von den Darstellern ausgehen und zu wenig Freiraum für den kreativen und wichtigen Arbeitsprozess lassen, in dem auch Umwege möglich sind oder zunächst einmal in die falsche bzw. andere Richtung gedacht werden kann und sollte (vgl. u. a. Riedel 2013: 32). Wenn schließlich ein grundlegendes Gesamtkonzept eines eigenen Produkts von den Schülern erarbeitet worden ist, kann unter Anleitung des Theater-Lehrers mit der Aufteilung von Rollen (aber auch der weiteren Aufgaben bei der Entstehung eines Theaterstückes) begonnen werden. Auch in diesem Prozess soll den Schülern auf der einen Seite deutlich werden wie komplex die Erarbeitung einer Produktion ist, aber auch welchen Gewinn die Auseinandersetzung mit Theater für sie bringt. Wichtig beim gesamten Entstehungsprozess ist, dass die Schülergruppe das Produkt als ihr eigenes ansieht und sich vollständig damit identifizieren kann. Die kreativen Kompetenzen, die sie dabei erwerben, können ihnen im weiteren Leben sicherlich in vielen Situationen dienlich sein.

\section{Praxisbeispiel aus der Deutschen Schule Valdivia in Südchile}

Wie eine mögliche Realisierung eines Theaterprojektes für den Bereich Deutsch als Fremdsprache aussehen kann, soll das Beispiel der Entstehung des Theaterstückes Es war 4 mal - Érase 4 veces verdeutlichen, das im Oktober 2013 an der Deutschen Schule Valdivia (Chile) zur Aufführung kam. Hierzu im Folgenden einige persönlichen Eindrücke:

Die Idee, Theater im Unterricht zu integrieren, entstand durch die Überlegung, wie man die Schüler mehr für das Deutsch-Lernen motivieren kann. Schließlich kam die Idee auf, in der 7. Klasse zwei der sechs Deutschstunden als Theaterkurs durchzuführen. Also vier Stunden Deutsch im Klassenzimmer und zwei Stunden Theater (Übungen, Kurzpräsentationen und ein Abschlussstück) in der Schulaula. Die Leitung der je zwei Theaterstunden übernahm ich, wobei die Deutschlehrer der drei Parallelgruppen dadurch indirekt eine schulinterne Fortbildung hinsichtlich der Theaterpädagogik erhalten sollten, da sie während des Theaterunterrichts anwesend waren. Die 7. Klasse bot sich an, da die Schüler am Ende der 6. Klasse die A2-Prüfung in Deutsch machen, die über den Schulverbleib entscheidet. Meist war die Motivation zu Beginn der 7. Klasse (auch vor dem Hintergrund der Pubertät) für das Erlernen der deutschen 
Sprache nicht sehr hoch, so dass viele Schüler kaum lernen wollten und die Leistungen in den Keller gingen. Meine Idee war also, dem entgegen zu wirken und ggf. durch das Theater ihr (gesprochenes) Deutsch zu verbessern sowie ihre kreativen Ausdrucksfähigkeiten zu fördern. Ich war im Vorfeld sehr gespannt, wie das Pilotprojekt von den Schülern angenommen werden würde und hatte im Laufe des Schuljahres teilweise arge Bauchschmerzen und Sorgen, da wir verpflichtend im Rahmen unseres Schulgeburtstages (155 Jahre Deutsche Schule Valdivia) am 4. Oktober ein Theaterstück aufführen sollten. Nach anfänglichen Übungen im 1. Schulhalbjahr, um die Schüler für die verschiedenen Aspekte des Theaters zu sensibilisieren, begannen wir dann Ende Juni mit den Vorbereitungen für die Aufführung.

Meine Idee war es, etwas zu Grimms Märchen zu machen, da in Deutschland 2013 das Grimm-Jahr gefeiert wurde und die Erstausgabe ihres MärchenSammelwerks vor 200 Jahren erschienen war, sozusagen Schulgeburtstag trifft Literaturgeburtstag. Das ganze bekam den Titel Es war 4 mal, da ich drei Deutschgruppen hatte und mein chilenischer Kollege Bruno Wersikowsky mit seiner Theater-AG ebenfalls von der Idee überzeugt war, eine Adaptation eines Märchens zu entwickeln, haben wir uns für ein bilinguales Projekt entschieden. Wir haben Schneewittchen und die 7 Zwerge ausgewählt, da hier von der Vorlage her schon viele Figuren bestehen und die größte der vier Gruppen 20 Schüler umfasste. Die Aufgabe an die Schüler war, dass sie das ihnen (auch aus der Muttersprache) bekannte Märchen nach ihren eigenen Ideen verändern sollten, so dass aber dennoch die Vorlage zu erkennen blieb. Wichtig war mir, dass die Schüler selbst ihre Stücke entwickeln. Genau wie die benötigten Dialoge, die wir teilweise aus Standbildern heraus improvisiert haben. Ich wollte, dass es ein Deutsch auf ihrem Niveau ist und nicht etwas, was sie auswendig lernen müssen und im Endeffekt nicht genau wissen bzw. fühlen, was sie eigentlich sagen. In einer Gruppe klappte dies sehr gut, die anderen beiden brauchten etwas mehr Unterstützung. Dennoch waren die entwickelten Geschichten zu ca. 80\% das Werk der Schüler. Außerdem war für meine drei Versionen wichtig, dass sie zwar auf Deutsch aufgeführt werden sollten, jedoch auch von einem Publikum $\mathrm{zu}$ verstehen sein mussten, das nicht deutschsprachig ist. D. h. die Bildkraft der einzelnen Szenen musste so eindeutig sein, dass die Gesamtgeschichte zu verstehen ist, ohne, dass man die Dialoge verstehen muss. Hier setzte dann meine Arbeit als Spielleiter ein, um von außen immer die Aussagekraft der Bilder im Blick zu behalten. Die einzelnen Arbeitsschritte waren im Überblick:

1. Märchen auf Deutsch vorlesen

2. Märchen von den Schülern als Gesamtgruppe auf Deutsch mündlich nacherzählen lassen

3. Märchen in sechs bis acht Szenen einteilen lassen

4. Sechs bis acht Szenen als Standbilder visualisieren

5. Wichtige Gegenstände, Personen und Hauptthema (Moral) herausarbeiten 
6. Ideen zur Verfremdung erarbeiten (in Kleingruppen auch in den Deutschstunden, da die Zeit fehlte)

7. Einigung auf jeweils eine gemeinsame Verfremdungsidee

8. Entwicklung des eigenen Märchens und Titelvergabe (auch in den vier weiteren Wochenstunden Deutsch)

9. Charakterisierung der Figuren (durch Improvisation und Zuordnung von Charaktereigenschaften)

10. Rollenvergabe (Überlegungen in Kleingruppen, eigene Wünsche, Ideen der Regie)

11. Erstellen einer Rollenbiografie

12. Malen der Bühnenbilder (im Kunstunterricht) [die im Endeffekt wegen fehlender Qualität doch nicht verwendet wurden]

13. Überlegungen zu Requisiten (teilweise auch im Deutschunterricht)

14. Vergabe von Aufgaben zum Besorgen der Requisiten (teilweise auch im Deutschunterricht)

15. Überlegungen zu Kostümen (teilweise auch im Deutschunterricht)

16. Proben in der Gesamtgruppe (zur regulären Theater-Unterrichts-Zeit)

17. Einzelproben mit Hauptfiguren (während der Geschichts- und Biologiestunden, da die Zeit fehlte)

18. Generalprobe (vor dem Abschlussjahrgang der Schule)

Das Ganze musste in einem Zeitraum von 15 Wochen geschehen, in denen jedoch drei Wochen Winterferien und eine Woche Nationalfeiertagsferien lagen, und ich effektiv nur 22 Unterrichtsstunden pro Gruppe hatte. Die Zeit drängte also sehr und zuletzt hatte ich schon fast die Hoffnung auf etwas Präsentables aufgegeben, was meinen Ansprüchen auch nur im Ansatz genügen würde. Hinzu kam die nicht immer leichte Arbeit mit bis zu 20 recht unruhigen Schülern auf Deutsch, also für sie in einer Fremdsprache. In den letzten Tagen vor der Aufführung mussten dann von Bruno und mir insgesamt über 50 Schüler koordiniert und mit Hilfe der Deutschkollegen und zwei deutscher Praktikantinnen beaufsichtigt werden. Die Proben verliefen viel chaotischer als ich es aus Deutschland gewohnt war. Es kamen zudem noch weitere Veranstaltungen in der Geburtstagswoche hinzu, was die Gesamtorganisation oftmals zusätzlich erschwerte, da beispielsweise der Aufführungsraum für Probenzwecke nicht zur Verfügung stand. Dennoch ist ein gutes Endprodukt entstanden, das mir gefallen hat, vor allem, weil ich mich sehr daran erfreut 
habe, wie sonst eher schwache und stille Schüler aufgeblüht sind und ihre Kreativität genutzt haben. Schüler, die Deutsch bis dato eher als unangenehmes Pflicht-Fach wahrgenommen haben, konnten sich verwirklichen und haben zum Teil sogar die etwas größeren Rollen gespielt. Alle Gruppen haben ihr Stück als eigenes Produkt angenommen, bei dem ich von außen nur die Stellschrauben justiert habe, sonst ihnen aber recht viel Freiheit gegeben habe. Dass nicht nur ich das so sehe, konnte durch eine abschließende Evaluierung bestätigt werden, in der die überwiegende Mehrheit der Schüler das Projekt als unbedingt wiederholenswürdig bewertet hat. Auch die sonstigen Beteiligten (mein Kollege Bruno, die drei Deutschlehrer der Gruppen, die zwei deutschen Praktikantinnen, die mich sehr unterstützt haben), die Eltern und vor allem die Kinder waren nach der Aufführung am 4. Oktober begeistert. Später erfuhr ich, dass sogar die Eltern der einen Klasse gemeinsam zur Premierenfeier in ein Restaurant gefahren sind, was die Klassenlehrerin sehr gefreut hat, da es zuletzt Spannungen unter den Eltern dieser Klasse gab und so der Theaterabend als Ausgangspunkt zur Verbesserung des Klassenklimas genutzt worden war.

\section{Inhalt der bilingualen Produktion Es war 4 mal - Érase 4 veces}

Es gab (wie oben erwähnt) vier Versionen des Märchens Schneewittchen:

\subsection{Schneewittchen und die 7 Personaltrainer}

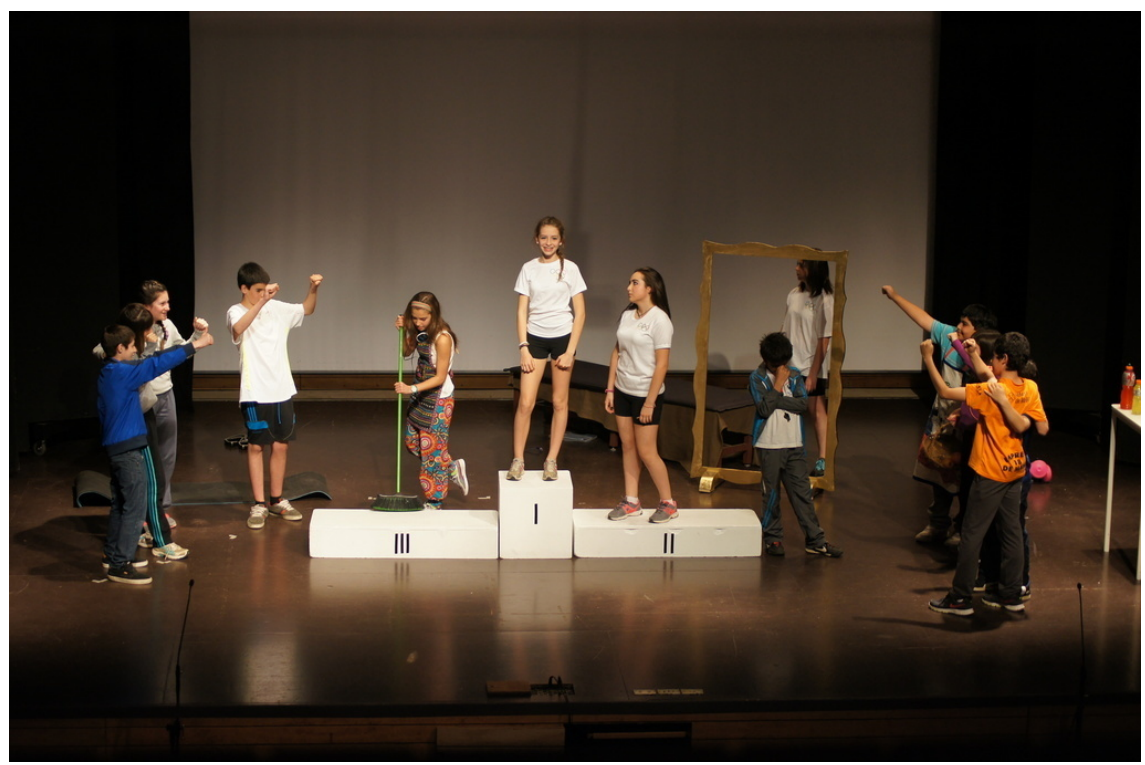

Abbildung 1: Abschlussszene aus Schneewittchen und die 7 Personaltrainer.

Eine Geschichte zweier konkurrierender Tennisspielerinnen vor dem OlympiaFinale. Die böse will die bessere (Schneewittchen) mit Hilfe ihres Trainers John 
vernichten. Er stellt Schneewittchen ein Bein, so dass sie verletzt ist und der Sieg in weite Ferne rückt. Da lernt sie die 7 Personaltrainer kennen, die ihr helfen wieder fit zu werden. Als das die böse Tennisspielerin über ihren magischen Spiegel erfährt, wird sie böse und lässt Schneewittchen mit Hilfe ihres Trainers und vergifteten Bananen töten. Die etwas zerstreute Putzfrau stößt beim Reinigen des Fitnessstudios der 7 Personaltrainer an die Massagebank, auf die die 7 Trainer das tote Schneewittchen aufgebahrt hatten. Schneewittchen fällt zu Boden und das vergiftete Stück Banane fällt aus dem Mund. Da dies just am Morgen des Finaltages passiert, schafft sie es noch rechtzeitig und besiegt ihre Kontrahentin Raquel.

\subsection{Schneewittchen und die 7 Freunde}

Zwei Schwestern leben in einer Villa am See. Die ältere denkt sie sei schön, aber ihr sprechender, allwissender Papagei sagt ihr, dass ihre kleine Schwester Schneewittchen viel schöner sei als sie. Josefa wird böse und benutzt den dümmlichen Gärtner, der in sie verliebt ist. Er soll ihre kleine Schwester umbringen. Schneewittchen kann jedoch entkommen und schwimmt auf die andere Seite des Sees, wo die 7 Freunde leben, die ihre Freizeit am Strand genießen. Sie kann bei ihnen bleiben. Pepe, der Papagei verrät Josefa, dass Schneewittchen bei den 7 Freunden weiterhin die Schönste am See sei. Wieder muss der Gärtner Peter herhalten. Als Eisverkäufer verkleidet soll er Schneewittchen am Strand vergiftetes Eis geben. Als diese davon nascht, fällt sie auf der Stelle um und kann erst vom Rettungsschwimmer Raúl reanimiert werden.

\subsection{Schneewittchen und die 7 Stylisten}

Auf einer Modenschau erfährt das ältere Model Elisabeth, dass ein junges Model namens Schneewittchen viel mehr Applaus bekommt und sie selbst vom Publikum kaum beachtet wird. Ihr Manager sagt ihr, dass Schneewittchen bereits viel mehr Likes auf Facebook habe als sie, daher befiehlt sie ihm, etwas zu unternehmen. Er bricht bei Schneewittchen ein, schneidet ihre Haare ab und verunstaltet sie während sie schläft. Schneewittchen bemerkt dies beim morgendlichen Blick in den Spiegel, erschrickt und läuft voller Panik um ihre Karriere einfach weg. Auf ihrer Flucht kommt sie zu einem Schönheitssalon. Da die Tür offen steht, geht sie hinein und probiert von den Cremes, der Schminke, dem Nagellack, legt sich auf die Massageliege usw. Die 7 Stylisten kommen gerade in ihren Salon, als Schneewittchen auf der Toilette ist. Sie bemerken, dass jemand von ihren Sachen genommen hat und treffen dann auf Schneewittchen, die ihnen erzählt, was passiert ist. Sie helfen ihr zu einer schönen neuen Kurzhaarfrisur. Schneewittchen darf im Salon bleiben und mithelfen. Sie begeistert die Kundinnen durch ihren neuen Look dermaßen, dass alle gern die gleiche Frisur haben wollen. Auch die Paparazzi werden erneut auf sie aufmerksam. So erscheint das beliebte Model wieder auf den 
Titelbildern der Hochglanzmagazine, was auch dem alten Model Elisabeth nicht entgeht. Sie schäumt vor Wut und wirft ihren Manager raus. Da sie wieder mehr Likes bei Facebook haben möchte als Schneewittchen, nimmt sie ihr Schicksal nun selbst in die Hand, bricht in den Schönheitssalon ein und hinterlässt für Schneewittchen eine vergiftete Creme. Schneewittchen denkt es sei ein Geschenk der Stylisten, probiert die Creme aus und stirbt. Erst der Kuss des vorbeikommenden Fußballstars, der sich schon auf der Modenschau in Schneewittchen verliebt hatte, lässt sie wieder erwachen.

\subsection{Schneewittchen und die 7 Tropfen Blut}

Die Geschichte ${ }^{2}$ ist als Anschluss an das bekannte Märchen zu verstehen: Die böse Stiefmutter ist mittlerweile alt und gebrechlich. Um wieder jung und fit zu sein und um länger leben zu können, verrät ihr Spiegel eine Lösung des Problems. Sie muss 7 Tropfen Blut der Tochter von Schneewittchen organisieren und dieses Blut trinken. Wie damals, als der Jäger Schneewittchen töten sollte, beauftragt sie nun wieder ihre Jägerin, die Tochter des ehemaligen Jägers, die Aufgabe zu erfüllen. Sollte dies erfolgreich sein, soll die Jägerin erfahren, wo ihr Vater ist. Die Tochter Schneewittchens ist mit der Tochter der Stiefmutter befreundet. Als die Jägerin gemeinsam mit ihrem Wolf die Tochter Schneewittchens findet und ihr das Blut abnehmen will, ahnt die Tochter der bösen Stiefmutter nichts Gutes. Daher rät sie der Jägerin lieber ihr, statt Schneewittchens Tochter Blut abzunehmen. Der Spiegel hatte dies genau so schon vorausgesehen und durch diesen Trick sich selbst befreit. Als die böse Stiefmutter also die 7 Tropfen Blut (ihrer eigenen Tochter) trinkt, verwandelt sie sich in ihr Spiegelbild, der Spiegel hingegen wird endlich befreit.

\subsection{Rahmenhandlung}

Die Rahmenhandlung des Gesamtstücks wurde durch einen bilingualen Dialog zwischen den Brüdern Grimm gestaltet, in dem Bruno auf Spanisch und ich auf Deutsch, das nicht-deutschsprachige Publikum ein wenig auf die Stücke vorbereitet haben. Außerdem hatten die Schüler hinter der Bühne so Zeit zum Umbau.

\section{Ausblick}

Da alles im Endeffekt doch so gut funktioniert hat und auch die Verantwortlichen in der Schule sehr zufrieden waren, wurde beschlossen auch 2014 erneut Theater auf Deutsch im Rahmen des Unterrichts der 7. Klassen anzubieten. Das erfolgreiche Theaterprojekt führte somit dazu, dass Theater fest in das DaF-Curriculum der Schule integriert wurde. Theater darf bzw. sollte nach

\footnotetext{
${ }^{2}$ Auf spanisch, entwickelt von der Theater-AG der DSV.
} 
diesen Erfahrungen in jedem Falle seinen Platz im DaF-Unterricht haben, da es positive Auswirkungen auf das (Deutsch-)Lernen der Schüler hat.

\section{Bibliografie}

Hoffmann, Tina (2008): Humor im Theater mit Fremdsprachenstudenten oder: Warum gerade Komödie? In: Hoffmann, Tina et al. (Hsg.) (2008): Humor. Grenzüberschreitende Spielarten eines kulturellen Phänomens. Göttingen (= Universitätsdrucke Göttingen), 205-214

Liebau, Eckart (2013): Rollenspiele zwischen Schule und Theater. Über Lehrerrollen und Schülerrollen im Schultheater. In: Schultheater 12, 38-40

Meisenberg, Norbert (2013): Unterwegs in fremden Welten. Figuren- und Rollenarbeit mit Grundschülern. In: Schultheater 12, 12-15

Oelschläger, Birgit (2004): Szenisches Spiel im Unterricht "Deutsch als Fremdsprache". In: gfl-journal 1, 24-34

Riedel, Klaus (2013): Occupy mich, Hamlet! Das Verhältnis von Rollenkonstruktionen und Dramaturgie. In: Schultheater 12, 30-35

Studt, André (2013): (K)eine Rolle spielen. Chancen und Probleme des Rollenbegriffs im Schultheater. In: Schultheater 12, 4-7 